\title{
HACIA LA INCORPORACIÓN DE LA ACCESIBILIDAD EN LAS FASES DEL DISEÑO CENTRADO EN EL USUARIO
}

\author{
Ana Paula Scariot, Luis Gerardo Montané Jiménez y Maria Del Carmen Mezura Godoy \\ Facultad de Estadística e Informática, Universidad Veracruzana, México
}

\begin{abstract}
RESUMEN
El Diseño Centrado en el Usuario (DCU) tiene como finalidad crear sistemas más interactivos, usables y adecuados a las necesidades de los usuarios, a través del uso de metodologías o modelos propuestos donde el usuario es la parte central del proceso de desarrollo. Tanto la usabilidad, como la accesibilidad pueden ser consideradas parte del DCU. Sin embargo, aunque la usabilidad es cada vez más incorporada en el diseño de sistemas, la usabilidad para personas con discapacidad visual aún puede mejorar, pues sigue siendo considerada deficiente para este tipo de usuarios. De este modo, este artículo presenta una reflexión de conceptos relacionados al DCU y metodologías que profundizan en la accesibilidad, discapacidad visual y usabilidad de los sistemas. Los resultados encontrados muestran que tradicionalmente la accesibilidad no es considerada en el proceso de desarrollo. Por lo tanto, en este artículo se propone de manera conceptual algunas fases que apoyan en la incorporación de la accesibilidad en las iteraciones de un proyecto tradicional hecho basado en metodologías ágiles.
\end{abstract}

\section{PALABRAS CLAVE}

Diseño Centrado en el Usuario, Accesibilidad, Usabilidad, Discapacidad Visual

\section{INTRODUCCIÓN}

Aunque hoy en día se tengan claras definiciones de discapacidad y accesibilidad, las personas con discapacidad aún tienen muchos obstáculos en la sociedad. Las políticas y normas relacionadas a esto no siempre consideran las necesidades de las personas con discapacidad o no hay seguimiento en su cumplimiento (OMS, 2011). Otro obstáculo es la falta de accesibilidad en los más variados aspectos, desde lo arquitectónico hasta los servicios en general. Con relación al aspecto tecnológico, la Organización Mundial de la Salud (OMS) informa que las personas con discapacidad, en comparación con las personas que no poseen alguna discapacidad, tienen tasas bajas de uso de tecnologías de información y comunicación, y en algunos casos es posible, incluso, que no puedan acceder a productos y servicios básicos como el teléfono, la televisión o Internet (OMS, 2011). Este es el escenario de mil millones de personas que viven con algún tipo de discapacidad, que es aproximadamente 15\% de la población mundial (OMS, 2011), considerando una estimación de 7 mil millones de personas. En este contexto, de manera más específica, la discapacidad visual actualmente es relevante porque aproximadamente 36 millones de personas son ciegas y 216.6 millones de personas poseen una discapacidad moderada a severa de la visión (Bourne et al., 2017).

Por la baja usabilidad que actualmente los Sistemas Interactivos (SI) ofrecen a las personas con Discapacidad Visual, se propone una reflexión sobre los conceptos relacionados al Diseño Centrado en el Usuario, usabilidad y accesibilidad, con un enfoque en las personas con discapacidad visual. También se presentan algunos modelos y métodos que pueden apoyar el proceso de diseño, además de una propuesta de diseño que considera la accesibilidad desde la concepción de un SI.

Estas temáticas se presentan en la siguiente estructura: la Sección 2 describe el Diseño Centrado en el Usuario (DCU), usabilidad y accesibilidad, además de una discusión de este escenario para las personas con discapacidad visual; la Sección 3 presenta un modelo para la creación de SI usables y accesibles; la Sección 4 presenta la conclusión y discusión final. 


\section{DISEÑO CENTRADO EN EL USUARIO}

El DCU, dentro del ciclo de vida de desarrollo de software, coloca el usuario en el centro del proceso, con todo su contexto y toda su interacción. Por esto, la psicología, sociología, ergonomía, ingeniería de software, mercadotecnia y otras áreas se relacionan para ofrecer Sistemas Interactivos más agradables y satisfactorios para las personas que los utilizan.

La ISO 9241:210 tiene un enfoque en el diseño y desarrollo de sistemas para que sean más utilizables, al enfocarse en el uso del sistema, con la aplicación de factores humanos/ergonomía y técnicas de usabilidad (ISO 9241:210, 2019). Los principios del DCU sirven para guiar a los profesionales durante el desarrollo de cualquier objeto, sistema, producto y/o artefacto. Estos principios son listados a continuación (Norman, 2018):

- Pensar en las personas: en todas las personas relacionadas con lo que se está desarrollando, identificando hasta su contexto de uso. Granollers (2003) añade que no se debe confundir "implicar al usuario en el diseño del sistema" con "realizar el diseño del sistema pensando en el usuario". Este último es el que debe ser utilizado.

- Considerar el "todo": no se debe considerar sólo el producto o sistema, pero si todo el contexto involucrado en el proceso, buscando encontrar el exacto problema a resolver, y su principal causa.

- Pensar sistemáticamente: en todas las piezas juntas para tener una mejor visión. Muchas veces, al dividir un proyecto en pequeñas partes, se pierde información crucial para un desarrollo bien hecho.

Norman (2018) añade que no se debe pensar en los detalles de uso de las herramientas. Lo más importante es pensar en el objetivo de las personas al utilizar el producto/servicio.

\subsection{Usabilidad}

La usabilidad es un término presente en el DCU definido por Nielsen (2012) como un atributo cualitativo que evalúa la facilidad de uso de las interfaces de usuario. Esto determina hasta qué punto un sistema, producto o servicio puede ser utilizado por usuarios para lograr objetivos con efectividad, eficiencia y satisfacción en determinado contexto de uso (ISO 9241:210, 2019). Desde hace casi una década, las mejores prácticas indican que se debe invertir por lo menos $10 \%$ del presupuesto del proyecto en usabilidad (Nielsen, 2012).

Hay muchas técnicas para desarrollar la usabilidad en un sistema. Estas pueden ser clasificadas en tres tipos: Inspección, Indagación y Test (Granollers, 2003), y siendo más conocidas como pruebas de usabilidad. Estas técnicas sirven para identificar mejorías en la usabilidad de los sistemas, generando resultados cualitativos y cuantitativos. Una evaluación comúnmente utilizada por expertos es la de Heurísticas de Nielsen (2012).

Sin embargo, las metodologías de desarrollo ágiles, como Scrum y Extreme Programming (XP), no la consideran en su proceso. Se centran más en la funcionalidad del sistema, de manera rápida y sencilla, sin considerar el aspecto humano de los usuarios. Incluso, esto es considerado un punto de dificultad en el área de la información, pues unificar el DCU con metodologías ágiles no es una tarea fácil, ya que involucra diferentes equipos de trabajo y, por lo tanto, diferentes mentalidades (Salah et al., 2014).

Hoy en día la usabilidad ha sido poco explorada en sistemas utilizados por personas con discapacidad visual. Nielsen (2001) realizó una prueba utilizando cuatro métricas de usabilidad: tasa de éxito, tiempo en la tarea, errores y calificación subjetiva, aplicadas a tres grupos de usuarios: personas que usan lectores de pantalla (principalmente usuarios que eran ciegos), personas que usan ampliadores de pantalla (usuarios con baja visión) y el grupo de control de usuarios sin discapacidades. Las cuatro métricas presentaron resultados menos favorables para las personas con discapacidad. Resaltando la calificación subjetiva, se ha verificado que la usabilidad es aproximadamente 3 veces mejor para usuarios sin discapacidad. Siebra et al. (2017) evaluaron 4 distintos sistemas comúnmente utilizados en Brasil. En 3 de ellos los usuarios con discapacidad visual tuvieron dificultades en realizar tareas, lo que también resulta en un nivel más bajo de usabilidad. La mejor evaluada fue el Facebook, incluso, porque sigue patrones de diseño de sistemas y de desarrollo, además de la preocupación social de su utilización.

\subsection{Accesibilidad y Discapacidad}

Con relación a la discapacidad, la OMS (2011) presenta la definición de discapacidad como deficiencias, limitaciones de actividad y restricciones en la interacción entre personas y factores personales y ambientales. 
Para disminuir aspectos negativos de la discapacidad, se debe ofrecer contextos accesibles, considerando factores ambientales y contextuales de las personas. Actualmente, el World Wide Web Consortium (W3C) es la organización con mayor iniciativa en cuanto a accesibilidad web y es llamada Web Accessibility Initiative (WAI). Ellos sugieren que la Web es diseñada para todos. Cuando la Web logra este objetivo, se torna accesible para individuos con una diversidad de habilidades auditivas, visuales, de movimiento y cognitivas (W3C, 2019).

WAI es considerado el estándar de accesibilidad en la Web, por la International Standard Organización (ISO), en la ISO/IEC 40500 (W3C, 2019), utilizando la guía Web Content Accesibility Guidelines (WCAG) 2.0. Sin embargo, sus reglas no son suficientes para mejorar el escenario de la usabilidad para personas con discapacidad. Lo mismo sucede con las iniciativas que surgieron en la Web y con las tecnologías de asistencia (lectores de pantalla, ampliador de pantalla, entre otras), donde la usabilidad para las personas con Discapacidad Visual necesita ofrecer un mejor nivel para alcanzar la inclusión con igualdad de oportunidades.

Esto ha creado un contexto favorable para la innovación en el proceso de diseño, con la creación de nuevas metodologías, como el Modelo (o Metodología) de Proceso de la Ingeniería de la usabilidad y la accesibilidad (MPIu+a); frameworks, como el de Silva et al. (2011); y sugerencias del DCU para personas con discapacidad, como las de Panchanathan et al. (2012). El MPIu+a considera tanto la usabilidad, como la accesibilidad, basado en tres pilares: ingeniería de software, prototipado y evaluación, considerando el usuario antes de todo y relacionando los tres pilares del método (Granollers, 2003). Sin embargo, su inclusión en las metodologías ágiles y en el ambiente laboral no es una tarea simple, debido a costumbres arraigadas en este ámbito.

Silva et al. (2001) generan un marco de referencia iterativo para la utilización del DCU, unificando conceptos del DCU con análisis de actividades y metodologías ágiles con las "historias de usuarios". Cada iteración es inspeccionada y corregida en la siguiente iteración. Sin embargo, este marco no contempla especificaciones sobre accesibilidad.

Panchanathan et al. (2012) sugiere que el DCU tenga tres dimensiones: la humana, la computadora y la interacción. Especialmente para los humanos, los autores sugieren considerar los conceptos relacionados a i) cognición; ii) las percepciones y sinapsis; y iii) las sensaciones, que son los tres niveles de la interacción humana. En su trabajo, los autores no consideran todo el proceso de desarrollo, pero toman en cuenta algunas sugerencias para el diseño. Por otro lado, Siebra et al. (2017) han identificado requisitos necesarios en la utilización de los dispositivos móviles para distintos tipos de discapacidad. Para la visual, ejemplos son de controles ajustables de brillo/contraste/color, proveer información de alertas de manera no visual, presentar ampliación sin prejuicios en el diseño de la página, entre otros.

\section{PROPUESTA DE FASES DEL DCU Y LA ACCESIBILIDAD}

Aunque actualmente el escenario de la usabilidad para personas con discapacidad está mejor que hace algunos años, aún se percibe que muchas empresas no consideran la accesibilidad como parte del proceso de desarrollo, por considerar más el desarrollo de funcionalidades que la utilización satisfactoria del sistema (Salah et al., 2014). Como solución para este ambiente de desarrollo tomado por las metodologías ágiles, se propone algunas fases (Figura 1) para lograr la accesibilidad y el entendimiento del usuario en el inicio de un proyecto ágil; y del DCU en cada Iteración.

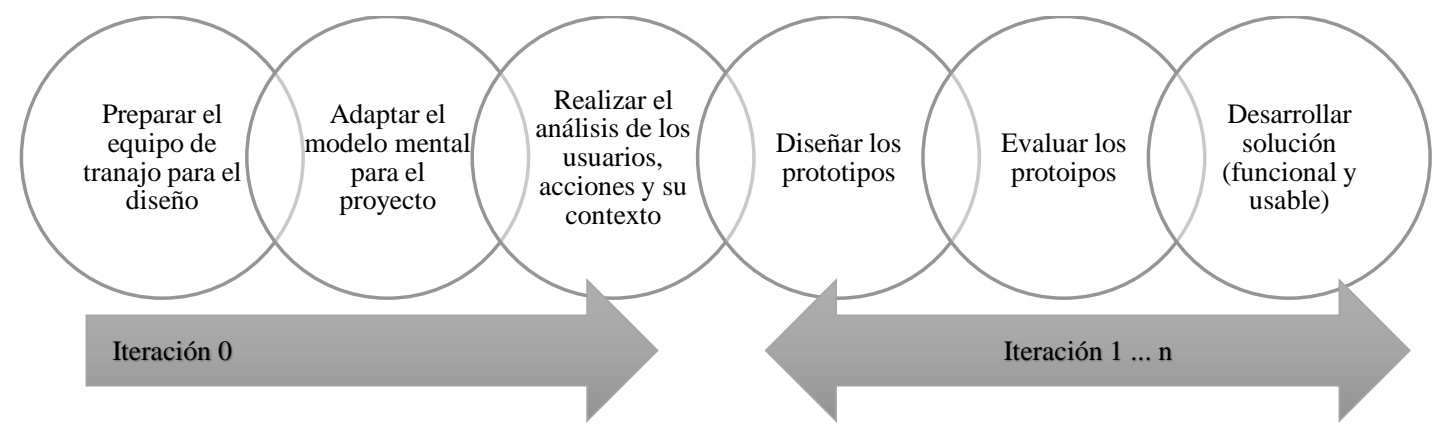

Figura 1. Fases del DCU para alcanzar la usabilidad y accesibilidad 
La iteración 0 es clave para lograr la adaptación del modelo mental, para considerar el usuario y sus necesidades durante todo el desarrollo en las siguientes iteraciones. En esta parte del proyecto, se sugiere el estudio de las reglas de usabilidad y accesibilidad adecuadas para el tipo de usuario, que puede ocurrir con la aplicación de una metodología como la MPIu+a (Granollers, 2003) y, profundizando para el código, de las prácticas de la W3C (2019). Si el equipo ya está involucrado en un entorno de desarrollo accesible, entonces, el análisis y entendimiento de los usuarios se considera suficiente.

Las otras iteraciones ocurren para la construcción de la solución de la manera ágil (ya tradicional en las empresas), aplicando la empatía por los usuarios obtenida en la fase anterior en todas las iteraciones. Otro punto es la inclusión de dos fases del DCU en cada iteración, que se refieren al diseño y prueba de los prototipos, con el debido refinamiento. El desarrollo debe considerar los requisitos de usabilidad y de accesibilidad percibidos en estas dos fases, junto a cada funcionalidad que será desarrollada.

\section{CONCLUSION}

Un contenido accesible ayuda en la inclusión de las personas con discapacidad, generando una mayor eficiencia en la realización de actividades en la web. Crear sistemas más accesibles resulta en una mejor experiencia de usuario para todos y no sólo las personas con alguna discapacidad. Por lo tanto, alteraciones simples que generan aplicaciones más amigables puede traer mejoras significativas para todos. Como solución, fueron propuestas algunas fases del DCU, con la demarcación de la usabilidad y de la accesibilidad en las pruebas y evaluaciones realizadas durante el desarrollo. Sin embargo, lo primordial es la preparación de un equipo de trabajo con una fase dedicada a su contextualización en un desarrollo inclusivo. Como trabajo futuro, se contempla probar el modelo propuesto en el desarrollo de un proyecto, aplicado a un equipo sin experiencia en el DCU, con un enfoque en la usabilidad y la accesibilidad.

\section{REFERENCIAS}

Bourne, R et al (2017). Magnitude, temporal trends, and projections of the global prevalence of blindness and distance and near vision impairment: a systematic review and meta-analysis. The Lancet Global Health, 5(9):e888-e897.

Granollers, Toni (2003). User Centred Design Process Model. Integration of Usability Engineering and Software Engineering. Proceedings INTERACT 2003.

ISO 9241:210 (2019). Ergonomics of human system interaction - part 210: Human-centered design for interactive systems. International Organization for Standardization, 2 edition.

Nielsen, Jakob (2001). Beyond accessibility: Treating users with disabilities as people. Recuperado de: https://www.nngroup.com/articles/beyond-accessibility-treating-users-withdisabilities-as-people/ en 28052020.

Nielsen, Jakob (2012). Usability 101: Introduction to Usability. Recuperado de: https://www.nngroup.com/articles/usability-101-introduction-to-usability/ en 27052020.

Norman, Don (2018). Principios del Diseño Centrado en el Usuario. Recuperado dehttps://www.nngroup.com/videos/principles-human-centered-design-don-norman/ en 29052020.

OMS (2011). Informe mundial sobre la discapacidad. Technical report.

Panchanathan, S. et al. (2012). Person-centered accessible technologies: Improved usability and adaptation through inspirations from disability research. Proceedings of the 2012 ACM International Workshop on User Experience in e-Learning and Augmented Technologies in Education, 1-6.

Salah, Dina et al. (2014). A systematic literature review for agile development processes and user centered design integration. In Proceedings of the 18th International Conference on Evaluation and Assessment in Software Engineering (EASE '14). NY, USA, Article 5, 1-10.

Siebra, Tatiana B. Gouveia, Jefte Macedo, Fabio Q. B. da Silva, Andre L M Santos, Walter Correia, Marcelo Penha, Marcelo Anjos, and Fabiana Florentin (2017). Toward accessibility with usability: understanding the requirements of impaired uses in the mobile context. In Proceedings of the 11th International Conference on Ubiquitous Information Management and Communication (IMCOM '17). Association for Computing Machinery, New York, NY, USA, Article $6,1-8$.

Silva, T. S et al. (2011). User-centered design and agile methods: A systematic review. Proceedings - 2011 Agile Conference, 77-86. https://doi.org/10.1109/AGILE.2011.24

W3C (2019). Introduction to web accessibility. https://www.w3.org/WAI/fundamentals/accessibility-intro/\#context. Accessed: 20191030. 